\title{
COVID-19 and Historic Influenza Vaccinations in the United States: A Comparative Analysis
}

\author{
Pranav Mirpuri ${ }^{1, *(1)}$ and Richard A. Rovin ${ }^{2}(\mathbb{D}$ \\ 1 Chicago Medical School, Rosalind Franklin University of Medicine and Science, \\ North Chicago, IL 60064, USA \\ 2 Department of Neurosurgery, Aurora Neuroscience and Innovation Institute, Milwaukee, WI 53215, USA; \\ Richard.rovin@aah.org \\ * Correspondence: pranav.mirpuri@my.rfums.org
}

Citation: Mirpuri, P.; Rovin, R.A. COVID-19 and Historic Influenza Vaccinations in the United States: A Comparative Analysis. Vaccines 2021, 9, 1284. https://doi.org/ $10.3390 /$ vaccines 9111284

Academic Editor: Nicolas Vignier

Received: 26 October 2021

Accepted: 3 November 2021

Published: 5 November 2021

Publisher's Note: MDPI stays neutral with regard to jurisdictional claims in published maps and institutional affiliations.

Copyright: (c) 2021 by the authors. Licensee MDPI, Basel, Switzerland. This article is an open access article distributed under the terms and conditions of the Creative Commons Attribution (CC BY) license (https:// creativecommons.org/licenses/by/ $4.0 /)$.

\begin{abstract}
The COVID-19 vaccination effort is a monumental global challenge. Recognizing and addressing the causes of vaccine hesitancy will improve vaccine uptake. The primary objective of this study was to compare the COVID-19 vaccination rates in US counties to historical vaccination rates for influenza in persons aged 65 and older. The secondary objective was to identify county-level demographic, socioeconomic, and political factors that influence vaccination rates. County level data were obtained from publicly available databases for comparison and to create predictive models. Overall, in US counties the COVID-19 vaccination rate exceeded influenza vaccination rates amongst those aged 65 or older $(69.4 .0 \%$ vs. $44 \%, p<0.0001) .2690(83.4 \%)$ of 3224 counties had vaccinated $50 \%$ or more of their 65 and older residents in the first seven months of the COVID-19 vaccination roll out. There were 467 (14.5\%) of 3223 counties in which the influenza vaccination rate exceeded the COVID-19 vaccination rate. Most of these counties were in the Southern region, were considered politically "red" and had a significantly higher non-Hispanic Black resident population (14.4\% vs. $8.2 \%, p<0.0001)$. Interventions intended to improve uptake should account for nuances in vaccine access, confidence, and consider factual social media messaging, especially in vulnerable counties.
\end{abstract}

Keywords: COVID-19 vaccination; vaccine hesitancy; SARS-CoV-2; coronavirus; demographic factors; socioeconomic status; political preference; race

\section{Introduction}

The COVID-19 pandemic vaccination roll-out is an unprecedented public health endeavor. The pandemic has swept the United States with over 670,000 dead and 41.9 million cases as of 20 September 2021 [1]. In fact, these official numbers are likely to be vast undercounts by many estimates due to rampant underreporting of cases and deaths [2]. Thus, with lives immediately at risk, it is crucial to appreciate barriers to vaccine access and to appreciate the important role of vaccine hesitancy.

Mandated, seasonal, and epidemic vaccination programs already exist in the United States [3]. The seasonal influenza vaccination program is well established and has decades of safety and efficacy data. Therefore, county influenza vaccination rates can serve as a standardized baseline of vaccine uptake. By comparing COVID-19 vaccination rates to those of influenza prior to the COVID-19 pandemic, county-level characteristics that influence vaccine uptake and hesitancy can be identified.

Comparing other vaccination rates to those of influenza is not without precedent. Nicholls et al. found in their comparison of influenza pneumococcal disease and shingles that interventions targeted towards older people would be more effective if they were vaccine specific and also emphasized the risks and benefits of vaccination to the individual and the community [4]. Similarly, Kwok et al. conducted a study on the association between influenza vaccination uptake with COVID-19 vaccination intention in nurses during the 
early phase of the pandemic [5]. They found that fewer than two-thirds of nurses intended to receive the vaccine when available.

In this study, we analyzed the influenza vaccination rates during the 2018-2019 influenza season and the COVID-19 vaccination rates through the first seven months of its availability using publicly available datasets. We specifically focused on persons 65 years of age and older as they are most at risk from COVID-19 and influenza disease severity and mortality; however, the overall COVID-19 vaccination rate across all ages was also analyzed [6,7]. Secondarily, we assessed the impact of county demographic, socioeconomic and political variables on COVID-19 vaccination rates to potentially identify at-risk populations and predictors for vaccination rates.

While our study can only provide evidence for correlation, this preliminary evidence can meaningfully inform future vaccination interventions which are vital in the setting of an urgent and evolving pandemic.

\section{Materials and Methods}

\subsection{Study Design and Outcome Measures}

This was a secondary analysis of publicly available data obtained from the Centers for Disease Control and Prevention (CDC) and from the County Health Rankings and Roadmaps (CHR\&R) program of the University of Wisconsin Population Health Institute. For a complete list of data sources and limitations of the county demographic variables, refer to Appendix A.

The primary objective of this study was to compare county vaccination rates for COVID-19 through the first seven months of eligibility and the 2018-2019 influenza season for residents aged 65 and older. The secondary objective was to determine how countylevel characteristics differentially influenced COVID-19 and influenza vaccination rates. "Rate" was defined as the percent of a county's population that is fully vaccinated for either COVID-19 or seasonal influenza.

We evaluated the following socioeconomic, political, and demographic variables:

Race-related variables: Percent of county population belonging to the following races: Nonhispanic White (NHW), Nonhispanic Black (NHB), and Hispanic.

Median Income: A continuous variable in dollars.

High School Completion Rate: Percent of county population who have completed high school. Literacy is a well-recognized mediator of health outcomes [8]. In the COVID-19 setting, it has been shown to predict susceptibility to misinformation [9].

Vaccine Hesitancy Estimate: Percent of county population who are estimated to be vaccine hesitant. The CDC derived the hesitancy rates by first estimating hesitancy on a Public-Use Microdata Area level using the Census Bureau's 2019 American Community Survey 1-year Public Use Microdata Sample (PUMA) and then on the granular county level through a PUMA-to-county crosswalk from the Missouri Census Data Center [10]. Vaccine hesitancy is complex and multifaceted; these survey-based estimates are subject to sampling error and nonresponse bias. However, since we use the hesitancy data in conjunction with actual vaccination rates and not to find geographic or temporal trends, the limitations of the dataset are somewhat mitigated.

Social Vulnerability Index (SVI): The SVI is a multidimensional metric for a community's ability to respond to adversity (like pandemic illness). It is created from census data and incorporates 15 factors representing socioeconomic status, household composition and disability, minority status and language, and housing type and transportation. An index value of 0 indicates little to no vulnerability, whereas a value of 1 indicates an extremely vulnerable county. The breadth of the measure makes it a uniquely powerful tool for comparison in our study. Several publications describe its use in identifying social vulnerabilities during disasters and have also qualitatively demonstrated its application [11,12].

COVID-19 Vaccination Coverage Index (CVAC): The CVAC is a measure that estimates the level of concern for difficulties in widespread COVID-19 vaccination coverage relating to supply and demand. The modular index is based on five themes including historic 
undervaccination, sociodemographic barriers, resource-constrained health systems, health accessibility barriers and irregular care-seeking behaviors. These themes are based on indicators such as proportion of individuals without insurance coverage, the proportion of individuals without visits to the doctor for routine checkup and the proportion of Medicare beneficiaries receiving the pneumococcal vaccine. Based on level of concern, the CVAC Index is categorized as follows: Very Low (0.0-0.19), Low (0.20-0.39), Moderate (0.40-0.59), High (0.60-0.79), or Very High (0.80-1.0). Although this measure has not been fully validated yet and there are limitations to its application, by aggregating baseline community characteristics it offers an interesting snapshot into the supply- and demandrelated problems that a county might face [13].

Segregation indices: these indices look at how evenly residents are distributed across a county's census tracts. We include both the Black vs. White index (segblk) and the non-White vs. White index (segnonwht). The residential segregation index ranges from 0 (complete integration) to 100 (highly segregated).

Internet access: Percent of households in a county with a broadband internet contract.

\subsection{Statistical Methods}

Parametric and non-parametric methods were used to compare the COVID-19 and influenza vaccinated cohorts. Comparisons were made based on county demographic, socioeconomic, and political data. Trends are described using Pearson's correlation. Multiple regression was used to identify predictors of vaccination rate. Statistical significance was $p \leq 0.05$. 95\% confidence intervals were provided where appropriate. Stata 15 (College Station, TX, USA) and R (Version 4.1.0) were used for statistical analysis.

\section{Results}

\subsection{COVID-19 and Influenza Vaccination Rates}

Data from 3224 US counties were available for analysis. For persons 65 and older, the median rate for COVID-19 vaccination was 69.4\% (Interquartile Range (IQR), 57.3-78.2\%) and $41.1 \%$ (IQR, 33-51.5\%) for all eligible ages. The median county level influenza vaccination rate for the 2018 season was $44 \%$ (IQR, 37-50\%).

We next determined the number of counties meeting a $50 \%$ or greater vaccination threshold. $83 \%$ of counties $(n=2690)$ achieved a COVID-19 vaccination percentage of $50 \%$ or greater for their 65 and older population. By contrast, when all eligible ages were included, only $16.5 \%$ of counties $(n=534)$ reached the $50 \%$ threshold. For the influenza vaccine, $31 \%$ of counties reached the $50 \%$ threshold for those 65 and older (Figure 1).

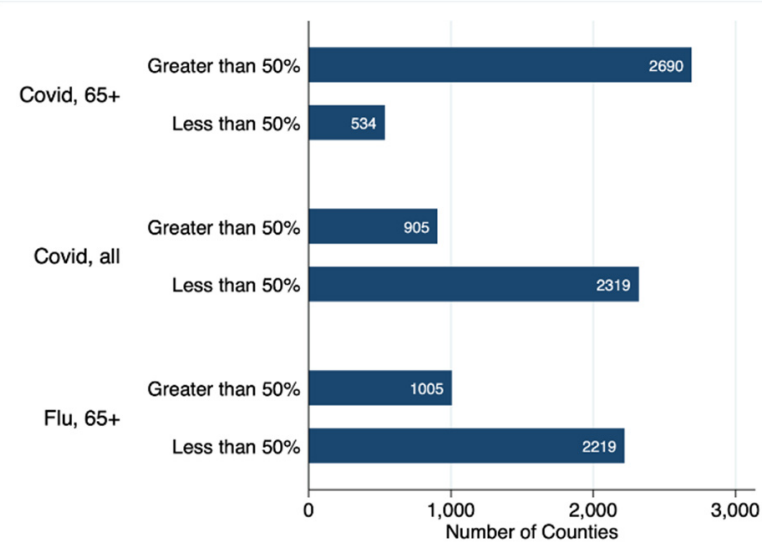

Figure 1. This bar graph shows the number of counties in the United States reaching the $50 \%$ vaccination threshold for COVID-19 vaccinations in the 65 and older population, COVID-19 vaccinations across all eligible ages, and for influenza vaccinations in the 65 and older population. 
Overall, there were five states with influenza vaccination rates higher than COVID-19 rates: Colorado, Georgia, Hawaii, Virginia, West Virginia (Figure 2). Taken together, in the first 7 months of the COVID-19 vaccine roll-out, acceptance was very high amongst persons 65 and older.
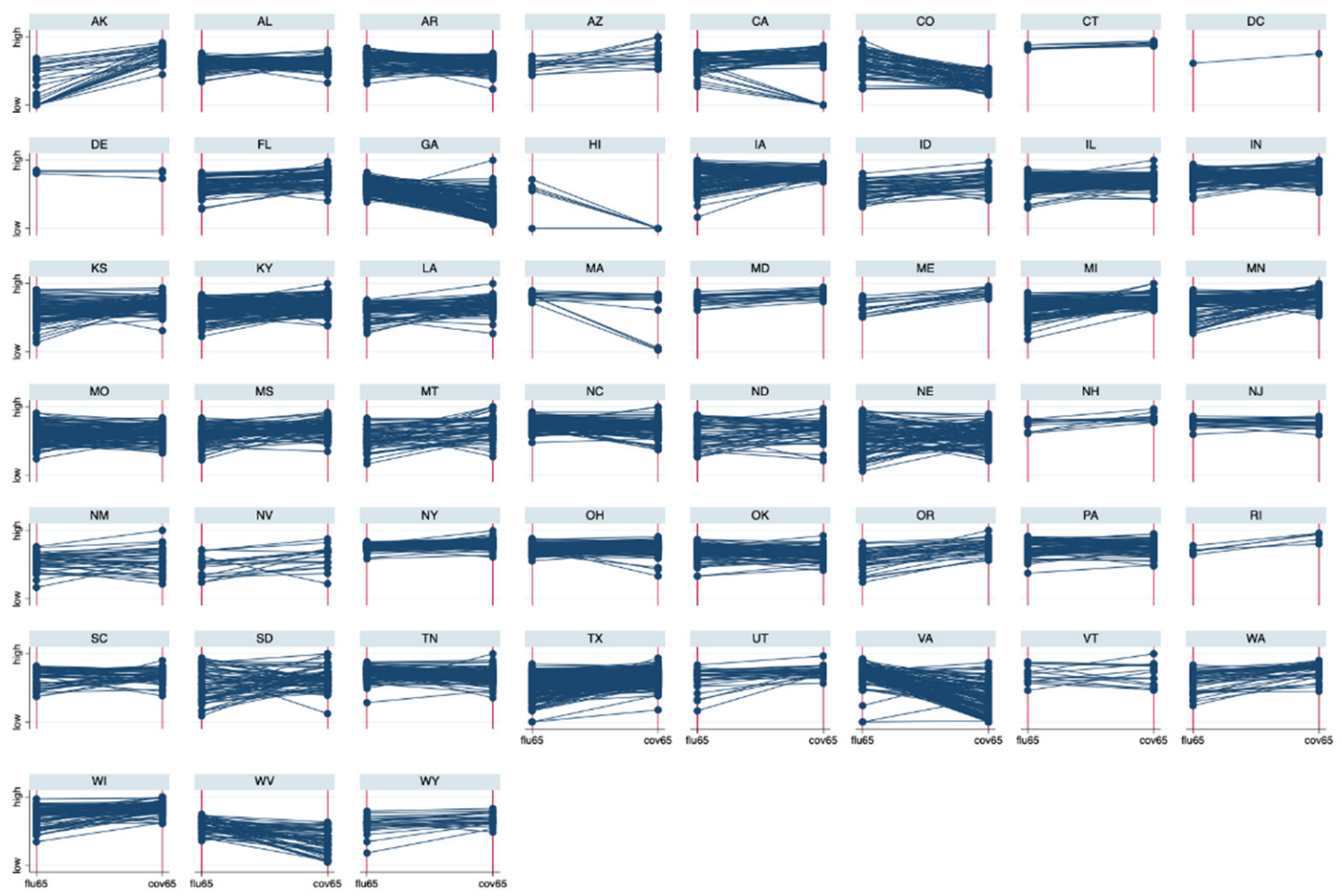

\section{Graphs by state}

Figure 2. Slopegraphs comparing influenza vaccination rates (left columns) to COVID-19 vaccination rates (right columns). Each line represents a county within a state or territory.

\subsection{County-Level Characteristics and Vaccination Rates}

We examined the influence that county-level demographic, socioeconomic, regional, and political features had on vaccination rates. Using Pearson's method, we found positive correlations between the rate of COVID-19 vaccination in the 65 and older population and the percent of NHW and Hispanic residents in a county $(0.09,0.13$, respectively, $p<0.0001)$. By contrast, there was a negative correlation between COVID-19 vaccination rate and the percent of NHB residents in a county $(-0.16, p<0.0001)$ (Figure 3 ).

For each county, we compared the COVID-19 vaccination rate to the influenza vaccination rate. There were 467 (14.5\%) counties with COVID-19 vaccination rates lower than influenza rates. We compared these "lowvax" counties to "highvax" counties for which the COVID-19 vaccination rates exceeded the influenza rates $(n=2756)$. Characteristics of the lowvax and highvax counties are presented in Table 1; highlights are summarized below. 

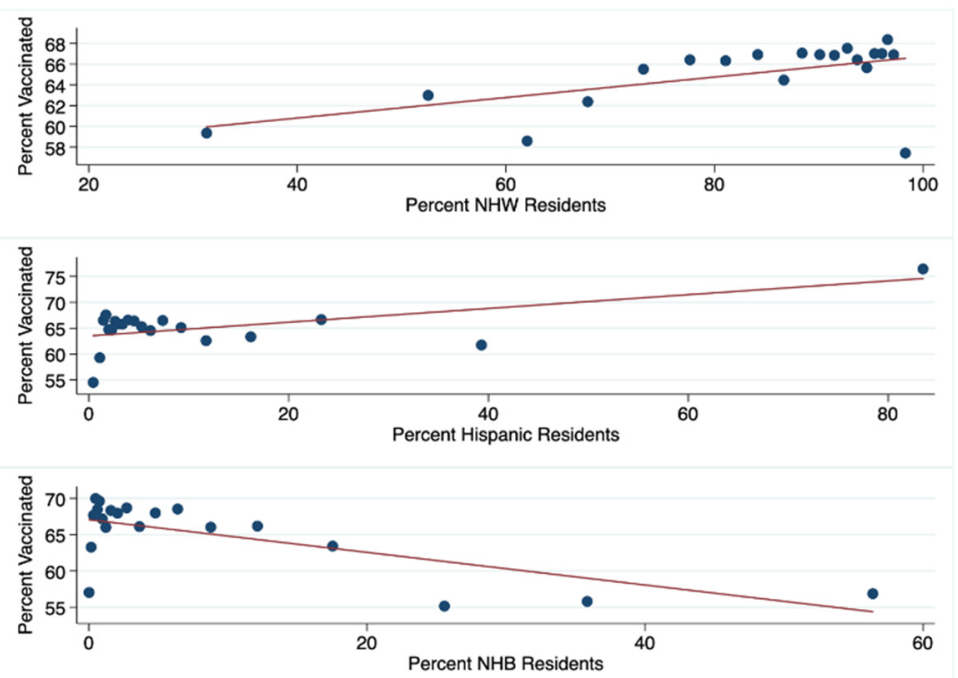

Figure 3. Binned scatter plots demonstrating the relationship between the rate of COVID-19 vaccination for the 65 and older population and the percent of county residents that are NHW, Hispanic, and NHB.

Table 1. Comparison of counties with COVID-19 vaccination rates lower (Lowvax) and higher (Highvax) than their influenza vaccination rates.

\begin{tabular}{|c|c|c|c|c|}
\hline & Total & Lowvax Counties & Highvax Counties & $p$-Value \\
\hline & $n=3223$ & $n=467$ & $n=2756$ & \\
\hline COVID-19 Vaccine, eligible ages & $41.10(33.00-51.50) *$ & $19.10(9.70-36.20)$ & $42.60(35.60-52.00)$ & $<0.001$ \\
\hline COVID-19 Vaccine, 65 and older & $69.37(57.37-78.20)$ & $28.50(13.70-45.20)$ & $71.20(62.30-79.10)$ & $<0.001$ \\
\hline Influenza Vaccine, 65 and older & $44.00(37.00-50.00)$ & $46.00(41.00-51.00)$ & $44.00(36.00-50.00)$ & $<0.001$ \\
\hline Percent NHB & $2.37(0.68-10.27)$ & $5.72(1.24-23.75)$ & $2.04(0.64-8.50)$ & $<0.001$ \\
\hline Percent NHW & $89.24(75.68-94.96)$ & $80.49(64.46-92.45)$ & $90.26(78.12-95.21)$ & $<0.001$ \\
\hline Percent Hispanic & $4.23(2.14-10.24)$ & $5.80(2.54-18.37)$ & $4.05(2.11-9.56)$ & $<0.001$ \\
\hline Segregation Index, White vs. Black & $32.37(0.00-50.29)$ & $18.06(0.00-36.52)$ & $34.84(0.00-51.74)$ & $<0.001$ \\
\hline Segregation Index, White vs. non-White & $27.83(15.91-37.47)$ & $18.81(0.00-30.75)$ & $29.07(18.50-38.33)$ & $<0.001$ \\
\hline Unemployment & $3.17(2.40-3.99)$ & $3.30(2.43-4.67)$ & $3.16(2.39-3.92)$ & $<0.001$ \\
\hline High School Completion & $34.42(29.67-39.15)$ & $32.90(28.00-38.38)$ & $34.62(29.93-39.28)$ & $<0.001$ \\
\hline Median Income (USD) & $49,491(41,865-57,333)$ & $43,918(35,096-55,439)$ & $\begin{array}{c}50,179.5 \\
(42,835-57,478.5) \\
\end{array}$ & $<0.001$ \\
\hline Uninsured & $9.04(6.10-12.54)$ & $9.74(6.35-13.21)$ & $8.93(6.07-12.45)$ & 0.17 \\
\hline Social Vulnerability Index & $0.5(0.25-0.75)$ & $0.49(0.24-0.8)$ & $0.5(0.25-0.74)$ & 0.85 \\
\hline Internet Access & $74(68-79)$ & $70(61-78)$ & 75 (69-79) & $<0.001$ \\
\hline Vaccine Hesitancy & $0.13(0.098-0.16)$ & $0.099(0.068-0.16)$ & $0.13(0.1-0.16)$ & $<0.001$ \\
\hline CVAC Index & $0.5(0.25-0.75)$ & $0.48(0.25-0.74)$ & $0.5(0.25-0.75)$ & 0.73 \\
\hline
\end{tabular}

${ }^{*}$ Median (IQR).

Overall, the median COVID-19 vaccination rate in the lowvax counties was $28.5 \%$ and $71.2 \%$ in the highvax counties $(p<0.001)$.

The lowvax counties had a significantly lower percentage (median) of non-Hispanic White residents $(80.5 \%$ vs. $90.3 \%, p<0.001)$, and significantly higher percentage of non- 
Hispanic Black $(5.72 \%$ vs. $2.04 \%, p<0.001)$ and Hispanic $(5.8 \%$ vs. $4.05 \%, p<0.001)$ residents compared to highvax counties.

Not only is the percentage of White and non-White residents significantly different between the low- and high-vax counties, their geographic distribution within counties also differs. The segregation index for White vs. Black residents is significantly higher in highvax counties compared to lowvax counties: 34.84 vs. $18.06, p<0.001$. The segregation index for White vs. non-White residents is also higher in highvax counties: 29.07 vs. 18.81, $p<0.001$. When we examined the correlation between segregation and COVID-19 vaccination rates, we found a strong negative correlation in lowvax counties for both White vs. Black and White vs. non-White indices: -0.35 and $-0.42, p<0.0001$, respectively. By contrast, in highvax counties, there was a positive correlation between segregation indices and the COVID-19 vaccination rates: 0.22 and $0.28, p<0.001$, respectively.

The social vulnerability index (SVI) was not significantly different for the lowvax and highvax counties: 0.49 vs. $0.5, p=0.85$. However, there were significant differences in several of the components of the SVI. For example, high school completion rate and median income were both higher in highvax counties, while unemployment was lower (Table 1).

The lowvax counties were predominantly in the southern region of the US and largely "red". In the Midwest, only 32 of 1055 counties were lowvax. All these counties were red. In the Northeast, 4 of 217 counties had a greater percentage of persons vaccinated for influenza than for COVID-19. All these counties were "blue". In the South, there were 276 of 1421 counties in which influenza vaccination percentage exceeded COVID-19 vaccination. Of these, 55 were blue, 221 were red. In the West, 73 of 418 counties were lowvax -32 were blue and 41 were red (Figure 4).

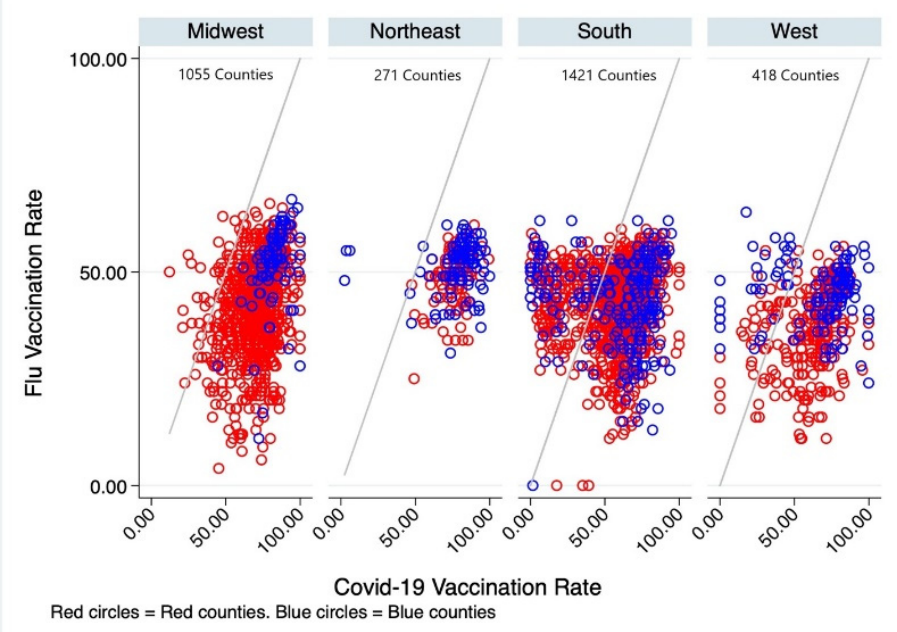

Figure 4. Influenza and COVID-19 vaccination in US regions and by political preference. The grey diagonal line represents equivalency of influenza and COVID-19 vaccination rate. Each circle represents a county. In counties above the diagonal line, the influenza vaccination rate exceeds theCOVID-19 vaccination rate. The counties below the diagonal line have COVID-19 vaccination rates that exceed influenza vaccination rates. Blue circles represent counties that voted for President Biden. Red circles represent counties that voted for President Trump.

There were differences in vaccination rates based on political affiliation. The mean COVID-19 vaccination rate for the 538 blue counties was $67.87 \%$ (95\% CI [65.77-69.98\%]), and it was $63.80 \%$, $(95 \%$ CI [63.08-64.52\%]) for the 2574 red counties. This difference is statistically significant, $p<0.0001$. Blue counties also had a greater mean influenza vaccination rate than red counties: $46.42 \%$ (95\% CI [45.60-47.24\%]) vs. $42.4 \%$ (95\% CI [42.01-42.78\%]), $p<0.0001$.

Regarding Internet access, there were differences between low- and high-vax counties. The median percent of households with any type of broadband internet access was sig- 
nificantly lower in the lowvax compared to the highvax counties: $70 \%$ vs. $75 \%, p<0.001$. Overall, as the percent of households in a county with internet access increased, the county's COVID-19 vaccination rate increased (Pearson correlation $0.21, p<0.0001$ ). Furthermore, as the percentage of households with internet access increased, the COVID-19 vaccination rate decreased in the lowvax counties (Pearson correlation $-0.31, p<0.0001$ ) but increased in the highvax counties (Pearson correlation $0.4, p<0.0001$ ). This is distinctly different than the findings for influenza vaccination. With increasing internet access, influenza vaccination rates overall, and for lowvax and highvax counties increased (Pearson correlation 0.4, $0.39,0.4, p<0.0001$, respectively) (Figure 5).

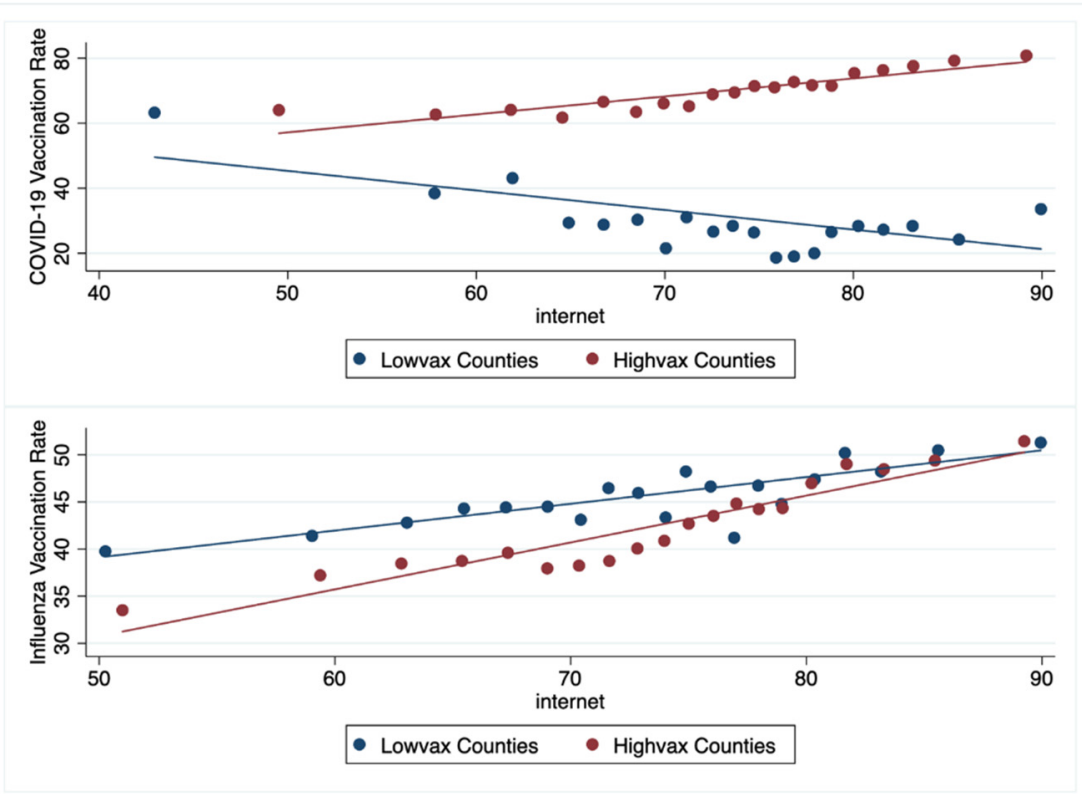

Figure 5. The association between Internet access and vaccination rates for low- and high- vax counties.

\subsection{Predictors of COVID-19 and Influenza Vaccination Rates in the 65 Years and Older Population}

Multiple regression was used to build predictive models. We first used the percent of residents 65 and older fully vaccinated for COVID-19 as the outcome variable. The predictive variables in Model 1 were the percent of NHW, NHB, and Hispanic residents in the county. In Model 2, the segregation indices were added. For Model 3, we added SVI. Model 4 included region and political affiliation. In Model 5, we added the CVAC index (Table 2). Models 1-4 were repeated for the outcome variable representing the percent of residents 65 and older fully vaccinated for influenza (Table 3).

Table 2. Multiple regression models to predict percent of county residents fully vaccinated for COVID-19.

\begin{tabular}{|c|c|c|c|c|c|}
\hline & Model 1 & Model 2 & Model 3 & Model 4 & Model 5 \\
\hline Percent NHB & $-0.262 * * *$ & $-0.278^{* * *}$ & $-0.303 * * *$ & $-0.171^{* * *}$ & $-0.169 * * *$ \\
\hline Percent NHW & -0.051 & -0.013 & $-0.147^{* * *}$ & $-0.106^{*}$ & $-0.106^{* *}$ \\
\hline Percent Hispanic & $0.114^{* * *}$ & $0.192^{* * *}$ & $0.111^{* * *}$ & $0.170 * * *$ & $0.173^{* * *}$ \\
\hline \multicolumn{6}{|l|}{ Segregation Index } \\
\hline White vs. Black & & $0.041 *$ & $0.064^{* * *}$ & $0.070^{* * *}$ & $0.070 * * *$ \\
\hline White vs. non-White & & $0.304^{* * *}$ & $0.327^{* * *}$ & $0.259^{* * *}$ & $0.257^{* * *}$ \\
\hline Social Vulnerability Index & & & $-13.592 * * *$ & $-5.609 * * *$ & $-4.995^{* *}$ \\
\hline
\end{tabular}


Table 2. Cont.

\begin{tabular}{|c|c|c|c|c|c|}
\hline & Model 1 & Model 2 & Model 3 & Model 4 & Model 5 \\
\hline \multicolumn{6}{|l|}{ Region (Index = Northeast) } \\
\hline Midwest & & & & -1.161 & -1.022 \\
\hline South & & & & $-14.025^{* * *}$ & $-13.806^{* * *}$ \\
\hline West & & & & $-11.783^{* * *}$ & $-11.624^{* * *}$ \\
\hline Red County (Index = Blue) & & & & -1.283 & -1.179 \\
\hline CVAC Index & & & & & -1.055 \\
\hline Constant & $70.289^{* * *}$ & $57.146^{* * *}$ & $74.423^{* * *}$ & $76.304^{* * *}$ & $76.346^{* * *}$ \\
\hline$n$ & 3224 & 3224 & 3142 & 3111 & 3111 \\
\hline R-squared & 0.0415 & 0.1121 & 0.1372 & 0.2079 & 0.208 \\
\hline aR-squared & 0.0406 & 0.1107 & 0.1355 & 0.2053 & 0.2052 \\
\hline Degrees of Freedom & 3220 & 3218 & 3135 & 3100 & 3099 \\
\hline
\end{tabular}

Table 3. Multiple regression models to predict county influenza vaccination rates.

\begin{tabular}{|c|c|c|c|c|}
\hline & Model 1 & Model 2 & Model 3 & Model 4 \\
\hline Percent NHB & $0.140 * * *$ & $0.111^{* * *}$ & $0.173^{* * *}$ & -0.01 \\
\hline Percent NHW & $0.160 * * *$ & $0.177^{* * *}$ & $0.119^{* * *}$ & 0.021 \\
\hline Percent Hispanic & $-0.073^{* * *}$ & $-0.074^{* * *}$ & 0.007 & $-0.038^{* *}$ \\
\hline \multicolumn{5}{|l|}{ Segregation Index } \\
\hline White vs. Black & & $0.135^{* * *}$ & $0.137^{* * *}$ & $0.123^{* * *}$ \\
\hline White vs. non-White & & $0.075^{* * *}$ & $0.099 * * *$ & $0.079^{* * *}$ \\
\hline Social Vulnerabiliity Index & & & $-11.712^{* * *}$ & $-11.499 * * *$ \\
\hline \multicolumn{5}{|l|}{ Region (Index = Northeast) } \\
\hline Midwest & & & & $-2.175^{* *}$ \\
\hline South & & & & 0.435 \\
\hline West & & & & $-4.606^{* * *}$ \\
\hline Red Counties (Index = Blue) & & & & $-3.478^{* * *}$ \\
\hline Constant & $28.966^{* * *}$ & $21.785^{* * *}$ & $30.367 * * *$ & $45.707^{* * *}$ \\
\hline$n$ & 3142 & 3142 & 3142 & 3111 \\
\hline R-squared & 0.0412 & 0.1948 & 0.2562 & 0.2735 \\
\hline aR-squared & 0.0403 & 0.1936 & 0.2548 & 0.2711 \\
\hline Degrees of Freedom & 3138 & 3136 & 3135 & 3100 \\
\hline
\end{tabular}

After controlling for demographic, socioeconomic, and political variables, the greatest negative predictor for the percentage of residents fully vaccinated for COVID-19 was residing in a Southern county. In the fully adjusted model for influenza vaccination, the greatest negative predictor was the SVI: as it increased, the percent of county residents 65 and older fully vaccinated for influenza decreased (Figure 6). 


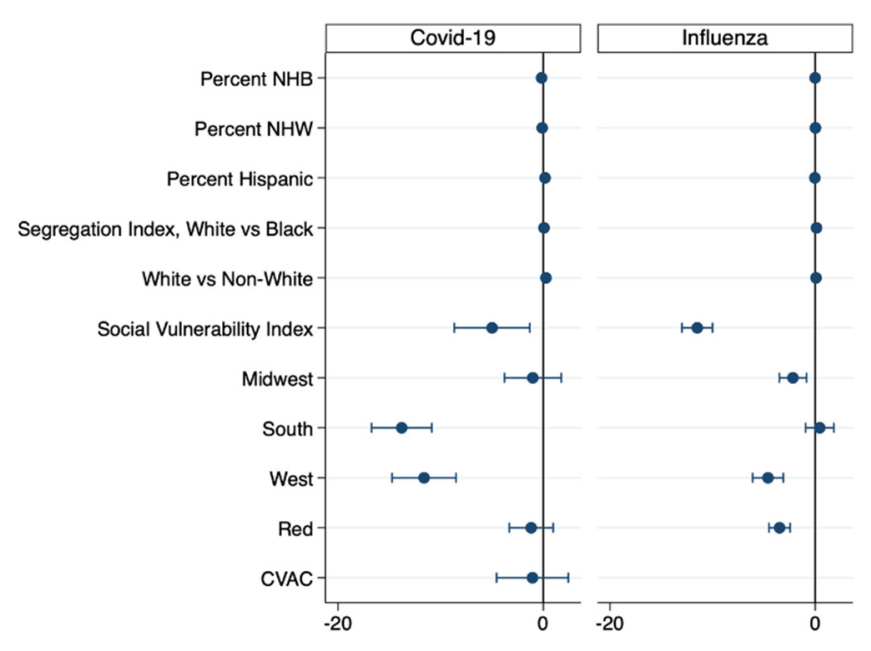

Figure 6. Coefficient plots for the fully adjusted multiple regression models of COVID-19 and Influenza vaccination rates. Horizontal bars represent 95\% CIs. Left of the 0 line (negative numbers) indicates lower COVID-19 vaccination rates.

\section{Discussion}

This study compared county level COVID-19 vaccination rates to seasonal influenza vaccination rates for the 65 and older population. In all but five states and a minority of counties, the COVID-19 vaccination rate exceeded the baseline influenza vaccination rate. In addition, far more counties reached the 50\% vaccination threshold for the COVID-19 vaccination than for the influenza vaccine (Figures 1 and 2). This suggests that acceptance of the COVID-19 vaccine was quite high in this population. This is not unexpected, as the push for and urgency of COVID-19 vaccinations has been unprecedented in size and scope, particularly in the 65 and older population [14].

We used US county seasonal influenza vaccination rates from the 2018-2019 season as a baseline to which we compared COVID-19 vaccination rates. We chose influenza vaccination as the comparator because of its long history, established infrastructure, and specific targeting of the 65 and older population. We found important differences between counties with a lower and higher COVID-19 vaccination rate than influenza. The lowvax counties were largely in the Southern United States, were politically "red", had higher unemployment, lower median income, lower high school graduation rate, and larger populations of people of color.

Our data also show that for the 65 and older population, as the percent of White or Hispanic residents in a county increases, the percent of those fully vaccinated for COVID-19 increases. By contrast, an increasing population of Black residents in a county is associated with decreasing COVID-19 vaccination rates (Figure 3). At first glance, this seems attributable to the black population's historic distrust in the medical establishment. There is literature to support this: Lin et al. found in their systematic review of COVID-19 receptivity that Hispanics reported higher or similar acceptance to White-Americans in contrast to Black-Americans, who reported heightened suspicion and lower confidence [15]. However, recent polling from the Kaiser Family Foundation shows that the percent of Whites, Blacks, and Hispanics who received at least one dose of a vaccine was essentially the same $(71 \%, 70 \%, 73 \%$, respectively). Also, a greater percentage of Whites $(14 \%)$ say that they will definitely not be vaccinated compared to Blacks (11\%) and Hispanics (9\%) [16]. As low confidence in the vaccine, then, is not the sole driver of this disparity, additional factors like vaccine access must be considered.

To that point, we found segregation was higher in highvax counties. And, as the segregation index increased, COVID-19 vaccination rates increased in highvax counties but decreased in lowvax counties. This suggests that people of color, particularly in 
lowvax counties, have greater barriers to vaccine access. This further suggests a solution: understand the geographic and cultural challenges populations of color face in accessing the COVID-19 vaccine, then plan the locations of distribution sites equitably $[17,18]$.

As alluded to above, vaccination success is mediated by vaccine hesitancy. Vaccine hesitancy is a complex psychosocial phenomenon [19]. As outlined by the 2015 SAGE Working Group, hesitancy consists of three factors: complacency, convenience and confidence [20]. Complacency exists when the perceived risk from the vaccine-preventable disease is low and therefore the vaccine is not regarded as important for personal health. Convenience is a function of availability, affordability, and the overall appeal of the vaccine. Confidence requires trust in health care professionals, science, and government [21]. In fact, vaccine hesitancy has been on the rise for several years, visible in crises such as the increased resistance to the MMR vaccine in Somali-Minnesotans due to a now discredited study linking vaccination to autism [22,23]. Indeed, The World Health Organization called vaccine hesitancy one of the 10 greatest global public health threats in 2019, even before the COVID-19 pandemic [24]. The literature also shows that hesitancy against one vaccine quickly transfers to others, condemning the COVID-19 vaccine to an uphill battle from the moment it received emergency use approval [25]. The COVID-19 vaccine has uniquely faced politicization and has been the subject of rampant misinformation [26]. The misinformation "infodemic" has obfuscated true data on the vaccine, instead playing to fears for safety, personal liberty, and governmental overreach.

Ye recently reported that the COVID-19 vaccination rate "is strongly associated with political partisanship", with vaccination rates in Republican counties lower than those in Democratic counties [21]. Our data are consistent with this political divide. Blue counties have significantly higher COVID-19 and influenza vaccination rates than red counties. As in the Ye paper, our data do not inform a causal mechanism. However, given the consistency of this association, specific messaging based on political party may enhance vaccination rates.

In a discussion of US vaccine hesitancy, the critical position of refugee, immigrant, and migrant (RIM) communities must also be considered in US vaccine hesitancy. It has been reported that RIM communities are disproportionately affected by COVID-19 and require special consideration in an equitable vaccination program [27]. Unfortunately, there is a paucity of literature on the specific drivers of vaccine hesitancy in RIM communities, most likely because RIM communities are not homogenous and vary significantly in factors such as socioeconomic status and cultural beliefs. Thus, it is recommended that vaccination efforts are undertaken in close collaboration with local partners who understand unique community-level factors. This allows for communities to be engaged directly to facilitate access to vaccines, lessen fear or mistrust of authorities, alleviate transportation challenges, and mitigate other barriers to widespread vaccination. For example, the use of portable immunization records has been suggested for migrant farmworkers, as they frequently relocate to find work, making it difficult to ensure continuity of care [28]. Interventions to alleviate vaccine hesitancy in other vulnerable communities in the US (for example, bringing vaccines to the people, messaging from trustworthy sources) can be applied to the RIM community with appropriate consideration to language and culture.

Lastly, the role of internet access in vaccine hesitancy cannot be overstated. Our data analysis highlights a compelling association between internet access and vaccination rates. For COVID-19, in highvax counties, increasing internet access is associated with increased vaccination rates, while in lowvax counties, increasing Internet access is associated with decreased vaccination rates. By contrast, for influenza, in both low- and high- vax counties, increasing internet access is associated with increased vaccination rates (Figure 5). This suggests a differential intensity of COVID-19 vs. influenza misinformation; it also suggests a differential susceptibility to COVID-19 misinformation in lowvax counties. But why are lowvax counties especially vulnerable to internet misinformation? Roozenbeek et al. found that people receiving information via social media were especially susceptible to COVID-19 misinformation and were more likely to resist vaccination [9]. It turns out 
that lower literacy, lower income, politically right-leaning, and self-perceived minority individuals are more susceptible to misinformation $[9,29,30]$. These are all hallmarks of lowvax counties.

\section{Conclusions}

Although this study cannot determine causation, the data showed a convincing picture of the impact of demographic, socioeconomic, and political factors on COVID-19 vaccine acceptance. Interventions intended to improve acceptance should focus on increasing access to and availability of the vaccine through community engagement, particularly in communities of color and in RIM communities. Factual and thoughtful social media messaging targeting vulnerable (lowvax) counties should also be considered.

Author Contributions: Conceptualization, P.M. and R.A.R.; Data curation, P.M. and R.A.R.; Formal analysis, P.M. and R.A.R.; Investigation, P.M. and R.A.R.; Methodology, P.M. and R.A.R.; Writingoriginal draft, P.M. and R.A.R.; Writing-review \& editing, P.M. and R.A.R. All authors have read and agreed to the published version of the manuscript.

Funding: This research received no external funding.

Institutional Review Board Statement: Not applicable.

Informed Consent Statement: Not applicable.

Data Availability Statement: The master dataset for this study has been archived in figshare https:/ / figshare.com/ (accessed on 22 October 2021).

Conflicts of Interest: The authors declare no conflict of interest.

\section{Appendix A}

Dataset Sources and Noteworthy Limitations

\section{COVID-19 Datasets}

COVID-19 County Vaccination Rates

- $\quad$ Obtained From: CDC COVID Data Tracker I Workbook: COVID-19 Vaccine in Texas (Dashboard).

- $\quad$ Source: CDC and Texas Department of Human Health Services.

- Limitations:

The COVID-19 Vaccination Rates are in flux as the vaccination effort is still actively underway. This is a preliminary research project aimed at identifying inequitable delivery of the vaccination and at informing interventions designed to decrease vaccine hesitancy.

- Reporting County-Level COVID-19 Vaccination Data I CDC

- California does not report the county of residence for persons receiving a vaccine when the resident's county has a population of fewer than 20,000 persons.

- Hawaii does not provide CDC with county-of-residence information.

COVID-19 Vaccine Hesitancy Estimates

- $\quad$ Obtained From: Vaccine Hesitancy for COVID-19 | Data I Centers for Disease Control and Prevention (cdc.gov (accessed on 20 September 2021)).

- Source: CDC.

COVID-19 Vaccination Coverage Index (CVAC)

- $\quad$ Obtained From: Vaccine Hesitancy for COVID-19 | Data I Centers for Disease Control and Prevention (cdc.gov (accessed on 20 September 2021)).

- Source: CDC in association with Surgo Surgo Precision For Covid.

- Limitation: All supply and demand related factors are not available at a granular county level consistently across all US counties. Indicators with more than $20 \%$ 
missing data across geographies were not included in the index. Instead, values were imputed with medial values across all counties for county level CVAC.

$$
\text { Social and Economic Factors }
$$

High school completion rate

- $\quad$ Obtained From: CHR\&R Program: County Health Rankings \& Roadmaps.

- Source: The American Community Survey (ACS) is a nationwide survey designed to provide communities with a fresh look at how they are changing.

Median Household Income

- Obtained From: CHR\&R Program: County Health Rankings \& Roadmaps.

- $\quad$ Source: The US Census Bureau, with support from other federal agencies, created the Small Area Income and Poverty Estimates (SAIPE) program to provide more current estimates of selected income and poverty statistics than those from the most recent decennial census.

- Limitation: "Receipts from the following sources are not included as income: capital gains; money received from the sale of property (unless the recipient was engaged in the business of selling such property); the value of income "in kind" from food stamps, public housing subsidies, medical care, employer contributions for individuals, etc.; withdrawal of bank deposits; money borrowed; tax refunds; exchange of money between relatives living in the same household; and gifts and lump-sum inheritances, insurance payments, and other types of lump-sum receipts" [31].

2018 Social Vulnerability Index

- Obtained From: CDC/ATSDR SVI Data and Documentation Download I Place and Health I ATSDR.

- Source: CDC/Agency for Toxic Diseases and Substances Registry (ATSDR).

- Limitations: Data is from 2018 and may not accurately represent rapidly changing counties. Use of census data only is a limitation as the census counts only where people live, not where they work or play [32].

Broadband Internet Access

- Obtained From: CHR\&R Program: County Health Rankings \& Roadmaps.

- Source: The American Community Survey (ACS) is a nationwide survey designed to provide communities with a fresh look at how they are changing.

Residential Segregation Index

- Obtained From: CHR\&R Program: County Health Rankings \& Roadmaps.

- Source: The American Community Survey (ACS) is a nationwide survey designed to provide communities with a fresh look at how they are changing.

- Limitation: The index was calculated only for counties with a black population greater than 100 persons.

\section{Clinical Care Factors}

Uninsured (\% of people under 65 without insurance)

- $\quad$ Obtained From: CHR\&R Program: County Health Rankings \& Roadmaps.

- $\quad$ Source: The US Census Bureau's Small Area Health Insurance Estimates (SAHIE) program produces estimates of health insurance coverage for all states and counties. SAHIE (census.gov (accessed on 20 September 2021)).

Influenza Vaccinations

- Obtained From: CHR\&R Program: County Health Rankings \& Roadmaps.

- Source: The Centers for Medicare \& Medicaid Services Office of Minority Health's Mapping Medicare Disparities (MMD).

- Limitation: This measure includes only the percentage of Medicare FFS enrollees who have received a flu vaccine and may potentially miss trends and disparities among younger age groups or people not enrolled in Medicare. 


\section{Political Affiliation \\ Democrat and Republican County Breakdown}

- Obtained From: GitHub—tonmcg/US_County_Level_Election_Results_08-20: United States General Election Presidential Results by County from 2008 to 2016.

- Source: 2020 election results published by Fox News, Politico and the New York Times.

\section{References}

1. CDC. COVID Data Tracker. Available online: https://covid.cdc.gov/covid-data-tracker (accessed on 20 September 2021).

2. Lau, H.; Khosrawipour, T.; Kocbach, P.; Ichii, H.; Bania, J.; Khosrawipour, V. Evaluating the Massive Underreporting and Undertesting of COVID-19 Cases in Multiple Global Epicenters. Pulmonology 2021, 27, 110-115. [CrossRef]

3. Williams, W.W.; Lu, P.-J.; O’Halloran, A.; Bridges, C.B.; Kim, D.K.; Pilishvili, T.; Hales, C.M.; Markowitz, L.E. Vaccination Coverage Among Adults, Excluding Influenza Vaccination-United States, 2013. MMWR Morb. Mortal. Wkly. Rep. 2015, 64, 95-102.

4. Nicholls, L.A.B.; Gallant, A.J.; Cogan, N.; Rasmussen, S.; Young, D.; Williams, L. Older Adults' Vaccine Hesitancy: Psychosocial Factors Associated with Influenza, Pneumococcal, and Shingles Vaccine Uptake. Vaccine 2021, 39, 3520-3527. [CrossRef]

5. Kwok, K.O.; Li, K.-K.; Wei, W.I.; Tang, A.; Wong, S.Y.S.; Lee, S.S. Influenza Vaccine Uptake, COVID-19 Vaccination Intention and Vaccine Hesitancy among Nurses: A Survey. Int. J. Nurs. Stud. 2021, 114, 103854. [CrossRef]

6. Zanettini, C.; Omar, M.; Dinalankara, W.; Imada, E.L.; Colantuoni, E.; Parmigiani, G.; Marchionni, L. Influenza Vaccination and COVID19 Mortality in the USA. medRxiv 2020. [CrossRef]

7. Keilman, L.J. Seasonal Influenza (Flu). Nurs. Clin. N. Am. 2019, 54, 227-243. [CrossRef]

8. Braveman, P.; Gottlieb, L. The Social Determinants of Health: It's Time to Consider the Causes of the Causes. Public Health Rep. 2014, 129, 19-31. [CrossRef] [PubMed]

9. Roozenbeek, J.; Schneider, C.R.; Dryhurst, S.; Kerr, J.; Freeman, A.L.J.; Recchia, G.; van der Bles, A.M.; van der Linden, S. Susceptibility to Misinformation about COVID-19 around the World. R. Soc. Open Sci. 2020, 7, 201199. [CrossRef]

10. Beleche, T.; Ruhter, J.; Kolbe, A.; Marus, J.; Bush, L.; Sommers, B. COVID-19 Vaccine Hesitancy: Demographic Factors, Geographic Patterns, and Changes over Time. Available online: https://aspe.hhs.gov/sites/default/files/private/pdf/265341/aspe-ibvaccine-hesitancy.pdf (accessed on 20 September 2021).

11. Wolkin, A.; Patterson, J.R.; Harris, S.; Soler, E.; Burrer, S.; McGeehin, M.; Greene, S. Reducing Public Health Risk During Disasters: Identifying Social Vulnerabilities. J. Homel Secur. Emerg. Manag. 2015, 12, 809-822. [CrossRef]

12. Flanagan, B.E.; Hallisey, E.J.; Adams, E.; Lavery, A. Measuring Community Vulnerability to Natural and Anthropogenic Hazards: The Centers for Disease Control and Prevention's Social Vulnerability Index. J. Environ. Health 2018, 80, 34-36.

13. Surgo Ventures. The U.S. COVID-19 Vaccine Coverage Index (CVAC) Methodology, Version 1. Available online: http:// precisionforcovid.org/cvac (accessed on 20 September 2021).

14. Copper, F.A.; de Vázquez, C.C.; Bell, A.; Mayigane, L.N.; Vedrasco, L.; Chungong, S. Preparing for COVID-19 Vaccine Roll-out through Simulation Exercises. Lancet Glob. Health 2021, 9, e742-e743. [CrossRef]

15. Lin, C.; Tu, P.; Beitsch, L.M. Confidence and Receptivity for COVID-19 Vaccines: A Rapid Systematic Review. Vaccines 2021, 9, 16. [CrossRef] [PubMed]

16. Kaiser Family Foundation (KFF). COVID-19 Vaccine Monitor Dashboard. KFF. 2021. Available online: https://www.kff.org/ coronavirus-covid-19/dashboard/kff-covid-19-vaccine-monitor-dashboard/ (accessed on 15 October 2021).

17. Hardeman, A.; Wong, T.; Denson, J.L.; Postelnicu, R.; Rojas, J.C. Evaluation of Health Equity in COVID-19 Vaccine Distribution Plans in the United States. JAMA Netw. Open 2021, 4, e2115653. [CrossRef]

18. Press, V.G.; Huisingh-Scheetz, M.; Arora, V.M. Inequities in Technology Contribute to Disparities in COVID-19 Vaccine Distribution. JAMA Health Forum 2021, 2, e210264. [CrossRef]

19. Schmid, P.; Rauber, D.; Betsch, C.; Lidolt, G.; Denker, M.-L. Barriers of Influenza Vaccination Intention and Behavior-A Systematic Review of Influenza Vaccine Hesitancy, 2005-2016. PLoS ONE 2017, 12, e0170550. [CrossRef]

20. MacDonald, N.E. Vaccine Hesitancy: Definition, Scope and Determinants. Vaccine 2015, 33, 4161-4164. [CrossRef]

21. Ye, X. Exploring the Relationship between Political Partisanship and COVID-19 Vaccination Rate. J. Public Health 2021. [CrossRef]

22. Bahta, L.; Ashkir, A. Addressing MMR Vaccine Resistance in Minnesota's Somali Community. Minn. Med. 2015, 98, 33-36.

23. Fridman, A.; Gershon, R.; Gneezy, A. COVID-19 and Vaccine Hesitancy: A Longitudinal Study. PLoS ONE 2021, 16, e0250123. [CrossRef]

24. Ten Health Issues. WHO Will Tackle This Year. Available online: https://www.who.int/news-room/spotlight/ten-threats-toglobal-health-in-2019 (accessed on 20 September 2021).

25. The Lancet Infectious Diseases. The COVID-19 Infodemic. Lancet Infect. Dis. 2020, 20, 875. [CrossRef]

26. Loomba, S.; de Figueiredo, A.; Piatek, S.J.; de Graaf, K.; Larson, H.J. Measuring the Impact of COVID-19 Vaccine Misinformation on Vaccination Intent in the UK and USA. Nat. Hum. Behav. 2021, 5, 337-348. [CrossRef] [PubMed]

27. Thomas, C.M.; Osterholm, M.T.; Stauffer, W.M. Critical Considerations for COVID-19 Vaccination of Refugees, Immigrants, and Migrants. Am. J. Trop. Med. Hyg. 2021, 104, 433-435. [CrossRef] [PubMed] 
28. Thomas, C.M.; Liebman, A.K.; Galván, A.; Kirsch, J.D.; Stauffer, W.M. Ensuring COVID-19 Vaccines for Migrant and Immigrant Farmworkers. Am. J. Trop. Med. Hyg. 2021, 104, 1963-1965. [CrossRef]

29. CDC Cases, Data, and Surveillance. Available online: https://www.cdc.gov/coronavirus/2019-ncov/covid-data/investigationsdiscovery/hospitalization-death-by-race-ethnicity.html (accessed on 20 September 2021).

30. Hawkins, R.B.; Charles, E.J.; Mehaffey, J.H. Socio-Economic Status and COVID-19-Related Cases and Fatalities. Public Health 2020, 189, 129-134. [CrossRef] [PubMed]

31. Census Bureau. 2010-2019 County-Level Estimation Details. Available online: https://www.census.gov/programs-surveys / saipe/technical-documentation/methodology/counties-states/county-level.html (accessed on 17 October 2021).

32. Flanagan, B.E.; Gregory, E.W.; Hallisey, E.J.; Heitgerd, J.L.; Lewis, B. A Social Vulnerability Index for Disaster Management. J. Homel. Secur. Emerg. Manag. 2011, 8. [CrossRef] 